\title{
Newsgames en Ecuador
}

Recibido: 31 de julio de 2018

Aceptado: 3 de septiembre de 2018

Publicado: 28 de noviembre de 2018

\author{
Isidro Marín Gutiérrez \\ imarin1@utpl.edu.ec \\ Universidad Técnica Particular de Loja (Ecuador) \\ Mónica Hinojosa Becerra \\ monica.hinojosa@unl.edu.ec \\ Universidad Nacional de Loja (Ecuador) \\ Javier Ruiz San Miguel \\ sanmiguel@uma.es \\ Universidad de Málaga (España)
}

Resumen: Se han creado videojuegos sobre temas políticos y sociales desde los inicios de este medio. En los últimos años, muchos desarrolladores informáticos están creando y lanzando juegos rápidamente en respuesta a eventos actuales. Estos juegos se conocen como "newsgames". Cada vez más el número de internautas aumenta utilizando la red para consumir noticias. Los newsgames podrían convertirse en una parte importante de cómo la gente entiende los acontecimientos actuales y ser un importante y expresivo género de videojuegos. El periodismo digital está haciendo que los medios busquen nuevas maneras de contar las noticias y de interactuar con su público. Un periodista debe de utilizar las tecnologías de la información y de la comunicación (TIC) y poner en práctica los nuevos formatos periodísticos. En Ecuador, este proceso tarda en arrancar, aunque ya algunos medios están poniendo en práctica estos formatos como es el caso de los newsgames. Nuestro trabajo estudia los newsgames que se están produciendo en Ecuador.

Palabras clave: Newsgames, serious games, videojuegos, periodismo digital, Ecuador.

1 Agradecemos a la Universidad Técnica Particular de Loja (UTPL), a la Universidad Nacional de Loja (UNL) y a la Universidad de Málaga por la ayuda prestada en la realización de la investigación, así como a la Universidad de Especialidades Espíritu Santo (UEES) y a su Facultad de Comunicación, que organizaron el I Congreso Internacional de Marketing, Publicidad y Comunicación (2018). Por último, agradecemos a los revisores del manuscrito y a sus aportaciones realizadas en la elaboración del texto final. 


\begin{abstract}
Video games have been created on political and social issues since the beginning of this medium. In recent years, many computer developers are creating and launching games quickly in response to current events. These games are known as "newsgames". Increasingly the number of Internet users increases using the network to consume news. Newsgames could become an important part of how people understand current events and could be an important and expressive genre of video games. Digital journalism is making the media look for new ways to tell news and interact with their audience. A journalist should use Information and Communication Technologies (ICT) and implement new journalistic formats. In Ecuador this process takes time to boot although some media are putting these formats into practice as is the case of newsgames. Our work studies the newsgames that are being produced in Ecuador.
\end{abstract}

Key words: Newsgames, Serious Games, Videogame, Digital Journalism, Ecuador.

\title{
1. Introducción
}

Los periódicos tradicionales han tenido una larga tradición de ofrecer a sus lectores rompecabezas y otra serie de juegos. El primer crucigrama en forma de diamante apareció en el periódico New York World en 1913 (Burton, 2005). A lo largo de la historia del rompecabezas impreso, la noción de que estos juegos de palabras son más que una distracción. En la edición en la que el Daily Mail de Gran Bretaña imprimió por primera vez un crucigrama, el periódico advirtió a sus lectores que los acertijos no son solo un juego, tienen valores educativos considerables (Engel, 1996).

Además de proporcionar un valor educativo general, algunos acertijos y juegos en los medios de comunicación requieren una comprensión de las noticias para completarlos con éxito. Los crucigramas basados en noticias se pueden encontrar en publicaciones de todo el mundo. Internet nos permite un papel activo en los juegos: al igual que con los acertijos, el uso de juegos (para complementar la información noticiosa) no deja de tener precedentes. Los medios de comunicación tradicionales tienen juegos interactivos de larga duración. Como se menciona líneas arriba, algunos de estos juegos tienen un elemento educativo, porque para jugarlos con éxito se requiere un conocimiento de los eventos actuales (Mancera Rueda, 2009).

Con el crecimiento de los medios de comunicación en Internet como una fuente de noticias preferida, los juegos basados en noticias tienen el potencial de ofrecer a su audiencia una forma adicional de explorar las noticias. A pesar de algunos obstáculos técnicos, los ciberperiodistas están en una ventajosa posición para crear juegos basados en noticias que complementen y mejoren los conocimientos de sus lectores. El ciberperiodismo es exploratorio, inclusivo y centrado en el usuario. Sus técnicas de producción pueden incluir la creación de presentaciones interactivas. Pero para que los juegos sean documentos periodísticos, resulta necesario abordar varias cuestiones sobre cómo se pueden presentar las noticias en forma de juego (Gómez \& Navarro, 2013). Y de ahí surge la pregunta: ¿los juegos son un medio apropiado para las noticias? 
Los media han introducido diferentes lenguaje comunicacionales cada vez más dinámicos y flexibles en cuanto a su forma de consumo. Cada vez más existen sitios y aplicaciones web para teléfonos inteligentes que consiguen una mayor comunicación. Los comunicadores han encontrado en los videojuegos una manera más eficiente de difundir noticias. Estos videojuegos se convierten en una excelente herramienta para informar a la población. Además, cada año la evolución de los videojuegos dentro de las industrias culturales y creativas en el mundo van a más (Arrobo et al., 2017, p. 103).

La prensa y la televisión se han visto desgastados y cada vez más pierden protagonismo entre los "millennials" o Generación Y, quienes pasan más el tiempo jugando con las videoconsolas que leyendo un libro o un periódico. Así, "los videojuegos son una de las tantas maneras en las que Internet ha cambiado en una generación de gente joven, la forma de socializar y percibir el entretenimiento" (Anneta, 2008, p. 230). El futuro del periodismo debe de estar centrado en adaptarse a las TIC y responder a las demandas de una nueva generación que será mayoritaria en un futuro.

\section{Juegos documentales}

Aquí separamos dos grupos de juegos. El primer grupo de estos se nutre de información de noticias actuales o históricas como fondo de un juego. En cambio, los del segundo grupo explican una historia de noticias a través de un juego. Vale la pena subrayar esta distinción, ya que influye tanto en el estilo del juego como en el tipo de información que se comunica. Una serie de videojuegos incluyen y usan ambos elementos, mientras que otros emplean el primer elemento hasta cierto punto.

Muchos juegos comerciales usan eventos históricos como telón de fondo. Por ejemplo, Return to Castle Wolfenstein (Raven, 2001), Medal of Honor: Allied Assault (Wolpaw, 2001), Brothers in Arms: Road to Hill 30 (figura 1) y Rome: Total War (Creative Assembly, 2004). Las referencias históricas que estos juegos hacen son más notables en el contexto del juego: los escenarios, la elección de las armas, los tipos de enemigos, el uso del lenguaje y las animaciones están diseñadas para referirse a un lugar y un momento histórico en particular. Para muchos de estos juegos, esta apariencia superficial es donde termina su afiliación con la recreación histórica. Por más que se intente, no se puede precisar una referencia histórica a los zombies y demonios que pueblan los pasillos del castillo Wolfenstein (López Pérez, 2013).

Algunos de estos juegos van más allá y muestran ubicaciones del mundo real para el juego. Uno de los logros más aclamados del diseño de niveles ocurre en el nivel de asalto a la playa de Medal of Honor: Allied Assault (Wolpaw, 2001), en el que el jugador "participa" en los desembarcos del Día D en la playa Omaha (Hess, 2007). De alguna manera, el jugador experimenta cierto realismo dentro del juego. Otros juegos llevan esta precisión histórica un paso más allá. Brothers in Arms: Road to Hill 30 (figura 1) no solo recrea partes de la campiña francesa de la década de 1940, sino que basa sus batallas en eventos reales y sus personajes en soldados reales que lucharon durante la invasión de Normandía (Hutchison, 2007). 
Figura 1: Portada del videojuego Brothers in Arms: Road to Hill 30.

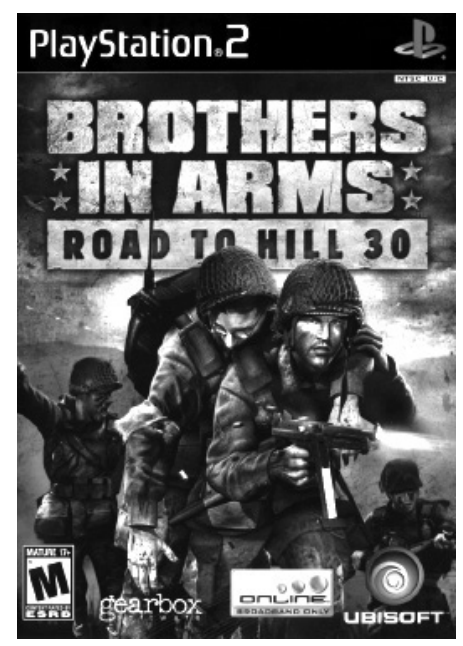

Fuente: Gearbox (2005).

Muchos de estos juegos emplean la precisión histórica que va más allá de ser un telón de fondo: echan mano de historiales o bases de noticias como una mecánica básica del juego. Por ejemplo, en un nivel muy básico, la mayoría de los juegos de disparos con temática de la Segunda Guerra Mundial, por ejemplo, Call of Duty (Infinity Ward, 2003) dan información práctica como cuántas balas solía llevar un arma de guerra en particular, así como una simple indicación sobre qué tan precisas debían disparar esas armas (Aarseth, 2007). Algunos juegos se amplían para incluir tácticas históricas de combate. En Brothers in Arms... (figura 1), el jugador debe dominar la táctica militar del mundo real de "disparar y maniobrar" para completar con éxito las misiones (Valderrama-Ramos, 2011). Así el juego Rome: Total War proporciona información visual sobre las buenas y malas tácticas del campo de batalla de la vida real (Squire \& Steinkuehler, 2005).

Sea al utilizar información histórica o de noticias como fondo, o mediante el juego real, estos videojuegos buscan generar una sensación de empatía entre el jugador y los personajes que participaron en los eventos reales en los que se basan los juegos. Varios videojuegos usan este sentido de empatía para realzar el drama del videojuego. En todo caso, como señala Frasca (2000), esta empatía es superficial, ya que la muerte del jugador (en el juego) está muy lejos de la muerte de un soldado de la vida real. Sin embargo, esto no impide que el jugador tenga una idea del contexto en torno al evento de noticias en cuestión.

\section{Adecuación de los juegos como forma de periodismo}

Varios autores cuestionan la conveniencia de los videojuegos como una forma de presentar noticias. El término "juego", en sí, sugiere una ligereza hacia el tema y una inherente inadecuación para temas difíciles, investigativos o sensibles (Domínguez Martín, 2014). 
Frasca (2000) ha explorado la posibilidad de "juegos serios" (serious games), es decir, juegos basados en noticias o información histórica. En ese sentido, prevé la controversia que se produciría si se hace un juego digital sobre el campo de exterminio en Auschwitz (Frasca, 2000). Cabe acotar el escándalo que rodeó el lanzamiento de uno de los primeros juegos para reclamar la etiqueta de "docu-game", JFK Reloaded (figura 2), donde el jugador asume el papel de Lee Harvey Oswald, quien disparó contra el presidente John F. Kennedy.

El videojuego trataba de esclarecer una pregunta que muchos documentales y otros medios periodísticos tradicionales han intentado responder: ¿fue asesinado John F. Kennedy por tres disparos de Lee Harvey Oswald desde un edificio? En lugar de examinar películas, tomar testimonios y examinar informes, el juego modela distancias, tiempos y balística para permitir al jugador determinar si era posible que Oswald tuviera los medios y la oportunidad de realizar el magnicidio (Fullerton, 2008).

Figura 2: Portada del videojuego JFK Reloaded.

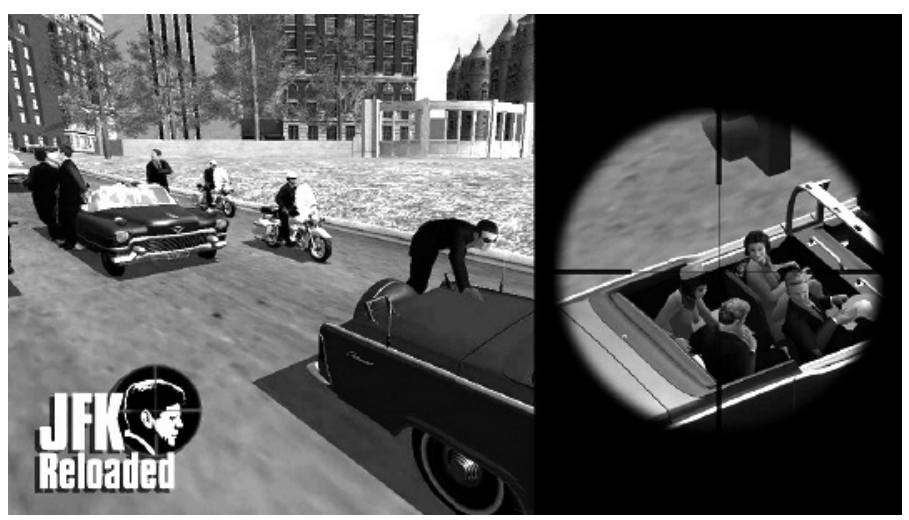

Fuente: Traffic Management (2004).

El propósito general del juego no es, como lo informaron algunos medios en ese momento, "disparar" al presidente Kennedy, sino explorar y, en última instancia, adherirse a un registro histórico. El juego recompensa al jugador por comprender los eventos noticiosos actuales de ese día. Para obtener un alto rango en el metajuego, el jugador no sólo debe entender los hallazgos básicos del Informe Warren (referido a la muerte del presidente), sino también familiarizarse con la atmósfera del juego. Obviamente, hay preocupaciones éticas bastante legítimas sobre el tema del juego; sin embargo, si separamos la controversia que rodea el juego de la entrega de la evidencia documental, entonces JFK Reloaded es bastante exitoso como documento periodístico.

En JFK Reloaded (figura 2) el videojuego es muy corto: el jugador tiene menos de un minuto para alinear su objetivo y disparar. Después de que termina el minuto de juego interactivo, el sistema analiza la capacidad del jugador para reconstruir el asesinato, según lo recopilado por el Informe Warren. El jugador recibe puntos según su adherencia al 
mencionado informe. Si bien el jugador casual puede saltarse estas "escenas cortadas", la única manera de "ganar" el juego es comprender cómo se llevó a cabo el asesinato (Raessens, 2006).

Algunos investigadores ven los juegos como un medio particularmente inapropiado para la transferencia de información de noticias. Existen autores que argumentan que la idea de recompensa y penalizaciones en los videojuegos es problemático para el periodismo, que valora la información como un fin, no como un medio (Domínguez Martín, 2014). La pregunta que sigue a esto es: ¿puede un videojuego tener información o conocimientos como producto final?

En Majestic (Anim-X, 2001) la información es el objetivo clave. En este juego, la búsqueda activa de información era, al menos, inicialmente el propósito principal del juego. El juego pedía a los jugadores descifrar las pistas que quedaban en las páginas web. Cada pista llevaría a más información, conduciéndose como una serie de minijuegos de resolución de acertijos. El videojuego se basó en el descubrimiento y la comprensión de la información. Una actividad clave en este tipo de videojuegos es la exploración. Para participar en el juego, los jugadores deben ser buscadores activos de información. Esta búsqueda activa de información también es una de las características de la audiencia del ciberperiodismo. Dicha dinámica ha cambiado la forma en que los ciberperiodistas hacen su trabajo.

Si la información se va a utilizar como el punto final principal para los juegos basados en noticias, y si el hallazgo de esa información se debe integrar en el juego, los productores de noticias deben tener mucho cuidado de establecer parámetros alrededor de la realidad construida que retratan. Al igual que con cualquier otro trabajo periodístico, debe hacerse una pregunta central: ¿qué tan precisa es la descripción de la realidad en los newsgames?

Las nociones de realidad y "realismo" son problemáticas en cualquier newsgame. Este es particularmente el caso en entornos interactivos que pretenden ser realistas, pero que nunca pueden ser totalmente precisos. Del mismo modo que la suma o la resta de una cita puede dejar una noticia tradicional abierta al sesgo, la suma o la resta de la geometría en un juego "realista" también puede generar un sesgo. También es importante tener en cuenta que las noticias en sí están mediadas y construidas. Existe un proceso editorial para la entrega de la mayoría de las noticias que implica la clasificación de información dispar para encontrar y presentar la información fáctica más relevante posible para el consumidor. Los juegos que presentan un "recorrido" sin interrupciones de una noticia o evento histórico, desde una perspectiva única (como la perspectiva en primera persona de muchos juegos), corren el riesgo de tergiversar o distorsionar los hechos sólo desde su perspectiva.

Ninguno de los videojuegos puede explicar completamente los eventos en los que se basa. Cada uno depende de la información de fondo suministrada o del conocimiento de los eventos por parte del jugador para explicar su significado en el problema con el que está relacionado. Los newsgames le dan un contexto al jugador: una opinión o una exploración de una noticia. 


\section{Periodismo en línea y juegos}

Una de las fortalezas de Internet es su capacidad de entretejer el contexto en torno al contenido. En ningún otro medio, tanto los creadores de noticias como los consumidores de noticias tienen un acceso tan fácil a los documentos de archivo y de fondo que los ayudan a examinar y analizar las noticias. Los ciberperiodistas tienen libertad para vincular material que ha sido fundamental para la formación de sus informes. Además, tienen una capacidad inigualable para hacer referencia a informes anteriores sobre el mismo tema y documentos creados por organizaciones e individuos con un interés en la noticia (Hinojosa, Robayo \& Maldonado, 2016).

Otra ventaja de la Internet es que el contenido puede tejerse y enhebrarse de la forma más apropiada. Mientras que los periodistas de medios tradicionales deben moldear las noticias para que quepan en un medio dado (como la televisión, radio o la prensa escrita), los ciberperiodistas, por el contrario, pueden elegir el tipo de medios que mejor se adapte a la historia que están informando. Un evento de noticias (con un fuerte elemento visual) puede ser reportado a través de galerías de videos o fotos, mientras que un problema complejo puede beneficiarse de la precisión y profundidad de los informes basados en texto o gráficos (Noci \& Salaverría, 2003).

En este contexto, cabe la interrogante: ¿qué propiedades tienen los juegos que podrían ayudar a comunicar noticias? Los videojuegos pueden educar, puesto que un usuario puede interactuar con la complejidad de un evento de noticias, tratando de replicar partes de él. Los juegos pueden permitirle a un jugador aprender sobre un conjunto de reglas o procedimientos tratando de lograr un objetivo mientras se adhiere a esas reglas.

Muchos sitios web de noticias ya emplean gráficos altamente interactivos para explicar un proceso o un conjunto de reglas. A menudo, estos gráficos no son lineales, lo que permite al usuario explorarlos en su tiempo libre y comprender la información dentro de ellos en sus términos. Si bien estos gráficos no son juegos, tienen similitudes, como estar centrados en el usuario y brindar al usuario la oportunidad de explorar una historia en sus propios términos. Los gráficos interactivos también se expanden en la noción de contexto. Muchos usos de las presentaciones interactivas en el ciberperiodismo se centran en la explicación de reglas y procesos o la ampliación de la información de fondo a las noticias importantes o persistentes (Valero Sancho, 2008).

Por supuesto, hay una clara diferencia entre las presentaciones interactivas y los videojuegos. Por ejemplo, no hay formas explícitas de "ganar" un gráfico interactivo como en la mayoría de los juegos. Sin embargo, hay ejemplos de presentaciones que difuminan las líneas entre los gráficos interactivos y los juegos. Son muy similares (en formato y contenido) a los gráficos de información, mientras que agregan un juego orientado a objetivos.

\section{Los primeros newsgames}

Los videojuegos son fundamentalmente un medio participativo donde la acción del jugador es de suma importancia. Esto los hace incompatibles, en muchos niveles, con la tarea 
periodística fundamental de informar sobre un evento de noticias que ya ha sucedido y no puede verse afectado por el jugador. Debido a esto, los juegos basados en noticias no pueden suplantar fácilmente a los informes de texto como el medio preferido para las noticias de última hora.

El valor de los medios interactivos, como los juegos, es agregar contexto alrededor de una historia, para explicar el contexto y el lugar de las noticias. Es importante destacar que este contexto puede transmitirse de una forma que puede ser educativa y atractiva para una audiencia que espera (cada vez más) que sus medios se adapten a ellos.

Prime Minister Forever (figura 3) le permite al jugador asumir el papel de contendiente político en procesos electorales tanto del Reino Unido, Canadá y Estados Unidos, como de Alemania o Australia. La interfaz que utiliza es muy similar a muchos de los gráficos de noticias producidos por medios de comunicación durante las elecciones. Sin embargo, en el juego, el jugador es libre de tomar sus propias decisiones sobre dónde y cómo hacer campaña. Para tener éxito, el jugador debe familiarizarse con los sistemas políticos.

Figura 3: Portada del newsgame Prime Minister Forever.

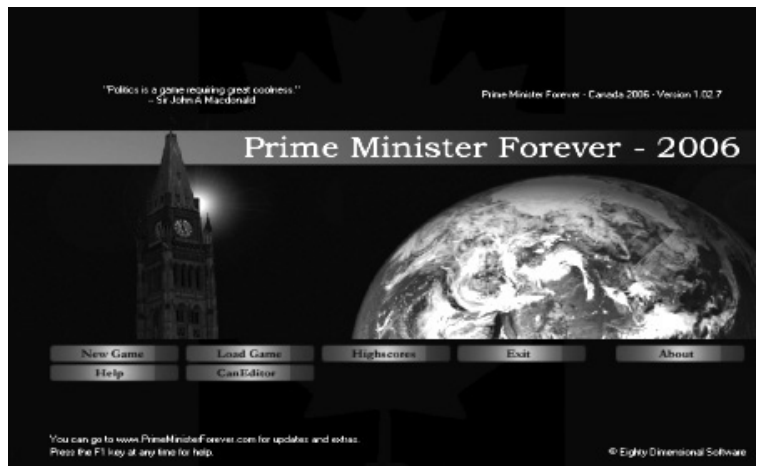

Fuente: Eighty Dimensional Software (2004).

Mientras el jugador estructura su propia campaña, se rige por las reglas de las elecciones de cada país. El jugador brinda márgenes de libertad en las acciones que se realiza en ese aspecto. Así, Prime Minister Forever proporciona al jugador una información general sobre los sistemas políticos. Este videojuego muestra cómo un juego de esta naturaleza podría funcionar como un complemento de ciberperiodismo.

Durante el 2008, la piratería frente a las costas de Somalia aumentó considerablemente: los ataques y secuestros de los buques mercantes estimuló la cobertura periodística de la piratería somalí. Un año después surgió el newsgame Cutthroat Capitalism (figura 4), donde el jugador asume el rol de un comandante pirata, con una inversión inicial de 50000 dólares, provenientes de los líderes tribales locales y de otros inversionistas. Su trabajo consiste en entrar en el mar con su equipo pirata, localizar y capturar barcos y negociar con éxito el rescate. 
El juego se convirtió rápidamente en una experiencia vivencial. El juego comienza con el mapa de la costa de Somalia (James, 2017). Nuestro barco está representado con un icono de un cráneo y parte de la ciudad de Eyl, un refugio pirata al norte de la capital del país, Mogadiscio. Uno puede ver los cargueros en el mapa con diferentes colores y se mueve a golpe del puntero hacia los objetivos. La intención es capturar estas naves y luego proceder a las negociaciones de rescate. Los procesos de negociación consiste en que el jugador elegirá un comportamiento ante los rehenes (alimentarlos, amenazarlos, agredirlos o matarlos) y una postura a tomar con las partes negociadoras (ser cordiales, erráticos o agresivos).

Se puede exigir hasta 30 millones de dólares. Así que el secuestro de una nave resulta ser un proceso tan metódico como comprar un coche. Las recompensas de los rescates se dividen entre el gobierno local, los líderes tribales, los inversionistas y la tripulación del barco pirata. Un jugador pierde principalmente por infravalorar lo capturado o por codicia. Este newsgame demuestra que los videojuegos pueden hacer buen periodismo, tanto como medio independiente de noticias y como complemento de las formas de cobertura (Carney, Eggertsson \& Doret, 2009).

Mientras que la infografía transforma los datos en bruto en gráficas visuales, el newsgame transforma esos datos en mecánica. Cutthroat Capitalism (figura 4) examina las estructuras del capitalismo global, donde los piratas son "parte" de este negocio. Para Harkin (2014), este juego develaría las dinámicas e injusticias de este sistema económico mundial, ya que coloca al jugador en el rol de pirata, quien tiene que "ganar" en la lógica económica mundial: es un cálculo de probabilidades donde uno debe de ganar dinero.

Figura 4: Portada del newsgame Cutthroat Capitalism.

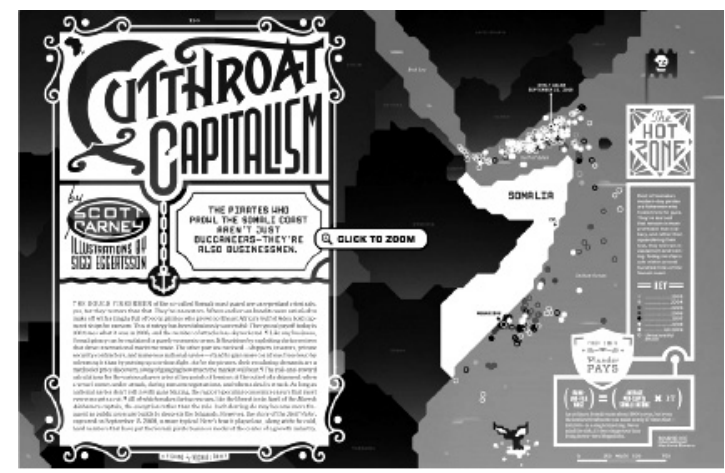

Fuente: Carney, Eggertsson \& Doret (2009).

El delicado estado financiero de los medios periodísticos actuales está promoviendo un cambio. Los ingresos por publicidad han caído en los últimos años. Los periódicos más modestos han tenido que eliminar personal o cerrar. Los periodistas no están de acuerdo en cuál será el nuevo formato para las noticias, pero sí coinciden en que los medios digitales desempeñarán un papel importante en su futuro. 
Sin embargo, los periódicos digitales aún siguen publicando las noticias de manera similar a los periódicos de papel. Incluso, si nos aventuramos un poco más, descubriremos que es un "copia-pega" del periódico entintado. Se suben segmentos de video como si fuera para televisión. La mayor parte de los podcast son documentos de la radio que se emiten a través de las ondas de radio. Las herramientas que hacen posible la creación y difusión de noticias se han vuelto sencillas, aunque el proceso de creación sigue siendo el mismo. Así, las noticias deben ser escritas y editadas por los periodistas (Archbold, 2014).

Los newsgames ofrecen una perspectiva estimulante acerca de los videojuegos. A diferencia de las noticias escritas para periódicos o reportajes editados para televisión, los videojuegos son programas informáticos en lugar de una forma digitalizada de los medios anteriores. Los newsgames muestran textos, imágenes, sonidos y videos, pero también hacen más cosas. Los newsgames simulan cómo se desarrollan los hechos mediante la construcción de modelos con los que los jugadores pueden interactuar. Los newsgames se convierten en una encrucijada entre los videojuegos y el periodismo. En este artículo, exploraremos las formas en que los videojuegos se han utilizado en las noticias actualmente (Bogost, Ferrari, \& Schweizer, 2012).

Frasca (2000 y 2009) realiza newsgames cortos, producidos de forma rápida y ampliamente distribuidos que tratan sobre acontecimientos actuales. Sus newsgames tratan de transmitir una opinión con el objetivo de persuadir a sus jugadores sobre un tema, pero bajo una perspectiva diferente. Frasca trata de lanzar el juego mientras la noticia siga siendo relevante en la agenda. Esto es todo un reto, ya que los juegos no pueden ser profundos ni complejos (Scolari, 2013). La mayoría de newsgames cubren historias aisladas de una manera corta y accesible; empero, son posibles tratamientos más largos y detallados de las noticias².

Así, el periodismo comprende un conjunto de valores y habilidades que el lector debe aprender de alguna manera. Es una alfabetización, un conjunto de reglas para leer, escribir y criticar un dominio particular del conocimiento. Los primeros pasos de la práctica del periodismo se toman tradicionalmente en las aulas o en los periódicos escolares, pero las cualidades de los videojuegos los convierten en medios complementarios ideales para la educación.

Es importante tomar en consideración a las comunidades de newsgames, que ofrecen una acción cooperativa. Al pensarse en un juego como The Sims, se asume que es un juego de asuntos privados, orientado al uso personal, sin estar conectado al exterior. Con las innovaciones recientes, los juegos se convierten en juegos masivos con multijugadores en línea (MMOG). Las nuevas plataformas soportan miles de jugadores simultáneos a la vez y estos jugadores están distribuidos en diferentes partes del globo. Los newsgames también utilizan esta tecnología (Chan \& Vorderer, 2006).

2 Vale la pena recordar que los juegos han sido parte de las noticias durante la pasada centuria, ya que las primeras sopas de letras aparecieron en The New York Times en 1913. En la década de 1920 aparecieron los crucigramas. Después de la II Guerra Mundial aparecieron en los periódicos los "puzzles" como la parte alegre y atractiva del día (Martínez Cano, 2016). 
A medida que la tecnología impacta en las noticias que se difunden, también cambia la forma de hacer periodismo. Los newsgames representan oportunidades inmediatas para las agencias de noticias, puesto que pueden ser la respuesta a los cambios que se vienen produciendo. Estos nuevos newsgames podrían complementar o reemplazar la cobertura actual de los hechos noticiosos. Los periódicos del futuro estarían integrados por la investigación, la redacción, la impresión, la distribución y la retroalimentación con el público. ¿Qué pasaría si las dinámicas de la corrupción en Ecuador pudieran llevarse a la práctica en el juego similar al Grand Theft Auto?

La revista digital Wired ha mostrado como se pueden integrar los newsgames en los artículos, demostrando la viabilidad de producir nuevos y diferentes productos para los medios de comunicación (Gómez \& Cabeza, 2016). El periodismo puede adoptar nuevas formas de concebir y de producir las noticias. Si bien los newsgames no son un bálsamo encantado que curará los males que aquejan a las agencias de noticias y a los periódicos tradicionales, pueden representar una oportunidad real y viable para ayudar a los ciudadanos a formarse ideas y tomar decisiones con respecto a la problemática actual.

\subsection{Hacer videojuegos y periodismo a la vez}

El juego ${ }^{3}$ es necesario para el desarrollo del ser humano. Apoya en el aprendizaje de roles y ayudan a los usuarios a comprender un poco más su mundo. En las sociedades arcaicas, se jugaba a "cazar" y en nuestras sociedades contemporáneas se juega a los “médicos". Así "en el juego, hay algo que se pone en juego que trasciende los límites inmediatos de la vida y que imparte cierta significación a la acción” (Huizinga, 1949, p. 93).

En la actualidad, algunos videojuegos se conciben más allá del entretenimiento. Pueden servir para educar, informar y concienciar sobre lo que sucede en el mundo. No es lo mismo leer un reportaje sobre la crisis económica en España, por ejemplo, que ser parte de un juego donde se asume el rol de una persona que recurre a una hipoteca para comprar una casa y quedarse luego sin empleo. Tampoco es lo mismo hablar de la situación de los inmigrantes que llegan a las costas de Europa en patera que asumir el rol de uno de estos en un juego y cruzar el mar Mediterráneo en una pequeña barca saturada de gente y sin GPS.

Los juegos de noticias (newsgames) nos permiten tener un rol y un estatus, experimentándose lo que sucede en distintas situaciones y así entender, de una manera más profunda, la actualidad. Las personas retenemos y nos conmovemos más según el grado de cercanía a la noticia. Es complicado tener empatía con individuos desconocidos, de los cuales desconocemos su vida cotidiana. No obstante, al experimentar y al convertirnos (en un tiempo limitado) en protagonistas de los sucesos, hace que nos sintamos cercanos.

3 La palabra "juego" abarca una innumerable cantidad de posibilidades y dinámicas que le permiten a la humanidad realizar diferentes acciones y de obtener diferentes estatus. Con la irrupción de las computadoras en la década de 1950, se desarrollaron los primero y rudimentarios programas lúdicos. Fue en la década de 1970 cuando comenzó su evolución hacia formatos más complejos (Hinojosa, Ruiz \& Marín, 2015). 
Asimismo, los newsgames potencian habilidades en sus jugadores, tales como la colaboración, la comunicación, las habilidades sociales, la creatividad, el pensamiento crítico, la resolución de diferentes problemas, la productividad, el aprendizaje de diferentes idiomas, el liderazgo, la adaptabilidad o el emprendimiento. De esta forma, los newsgames enseñan, entretienen, crean conciencia y estimulan a sus jugadores (Paíno \& Rodríguez, 2016).

\subsection{Newsgames: contexto y retos del periodismo}

En la sociedad actual, en la "sociedad de las pantallas", la prensa tradicional está quedando rezagada. El número de consumidores de prensa está disminuyendo. Internet está cambiando los formatos tradicionales y creando nuevas formas de comunicación. Los sitios web van en aumento, incluso aquellos donde se crean noticias interactivas en conjunción con plataformas de videojuegos. Así, "la red Internet se ha convertido, más de diez años después de su comercialización, en una tecnología cotidiana de la vida y en el entorno de millones de personas en todo el mundo" (García \& Rupérez, 2007, p. 56). Los newsgames intentan responder a unas audiencias cada vez más complejas ${ }^{4}$. Las noticias tienen que satisfacer a un público variopinto que busca cada vez más el entretenimiento ${ }^{5}$.

La demanda actual se puede expresar de manera individual: el usuario de Internet no tiene necesariamente que informarse pasando por los contenidos diversos de un periódico, sino que puede escoger directamente un portal en línea (García \& Rupérez, 2007, p. 63).

La Unión Europea propuso una serie de objetivos para la comunicación, donde cada uno de sus integrantes se comprometía a fortalecer el desarrollo tecnológico encaminado al desarrollo de la cultura tecnológica. El debate se centra en definir cuáles son las competencias necesarias para todos en esta nueva sociedad del conocimiento (MarínGutiérrez, Díaz-Pareja, \& Aguaded, 2013).

4 Hay diferentes tipos de lectores de noticias: a) los que solo leen los títulos, b) los que prefieren periódicos más fáciles de leer, $c$ ) los que solo ven las fotografías o $d$ ) los que buscan infografías.

5 Actualmente, la comunicación, la información y el entretenimiento utilizan Internet como un canal común: “los juegos de consola conviven con las páginas de opinión" (García \& Rupérez, 2007, p. 71). 
Figura 5: Portada del newsgame Kabul Kaboom!

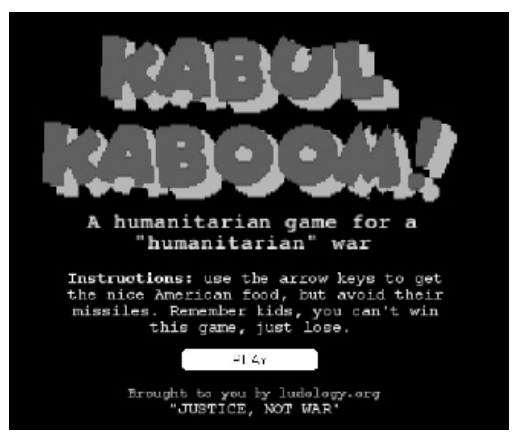

Fuente: Treanor, Mateas \& Wardrip-Fruin (2010).

A comienzos del milenio aparecen los primeros newsgames del uruguayo Gonzalo Frasca. En Kabul Kaboom! (figura 5) se adquiere el rol de una persona afgana que tiene que recoger cajas de ayuda humanitaria, la "comida americana saludable" (hamburguesas), mientras el ejército de los Estados Unidos bombardea constantemente. Al tener que sortear las explosiones, en Kabul Kaboom!, el jugador se convierte en una víctima del bombardeo estadounidense en dicha ciudad afgana. Así, este newsgame abordaba un tema noticioso, pero mucho más dinámico, donde el jugador puede empatizar con la acción que se desarrolla (Treanor, Mateas \& Wardrip-Fruin, 2010).

Figura 6: Portada del newsgame September 12th.

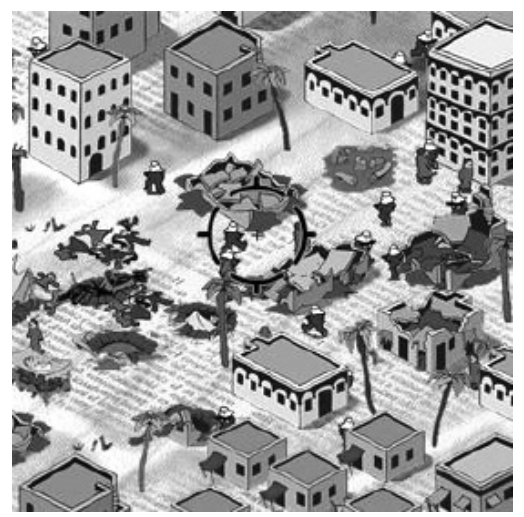

Fuente: Frasca (2009).

En el 2003, Frasca creó otro newsgame similar, llamado September 12th (figura 6), que tiene también a Kabul como escenario de operaciones. El jugador adquiere el rol de soldado estadounidense, quien debe identificar los objetivos terroristas y bombardearlos. ¿Cuál es el problema? Hay un poco de retraso en el tiempo y es casi imposible evitar "daños colaterales". Mientras más bajas civiles se registren, más aumentará el número de habitantes radicales en Kabul (Frasca, 2009). September 12th utiliza el conocimiento como 
su objetivo final. En su pantalla de título dice que "no puedes ganar y no puedes perder". No hay un "final" tradicional en este juego: el mensaje que Frasca plantea es que la guerra contra el terrorismo es esencialmente inviable.

Figura 7: Portada del newsgame Madrid.

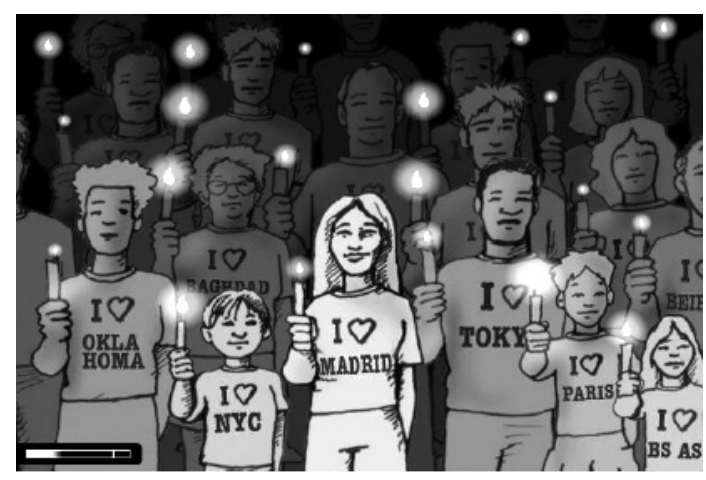

Fuente: Frasca (2009).

En el año 2004 Frasca creó Madrid (figura 7), referido al atentado terrorista del 11M. El objetivo de este newsgame era rendir homenaje a las víctimas de los atentados terroristas de Madrid. A diferencia de los anteriores newsgames comentados, Madrid fue desarrollado y publicado dos días después de producido el atentado. El newsgame consiste en avivar las llamas de las velas de los manifestantes. Se subraya la dimensión emocional del hecho, un aspecto que generalmente no está muy presente en los videojuegos (Frasca, 2009).

Posteriormente, se lanzaron más newsgames, tales como Democracy2 o Bacteria Salad (figura 8), ambos desarrollados por Ian Bogost. En este último, los jugadores cultivan verduras con fines de lucro y tratan de evitar el envenenamiento de los consumidores. En estos newsgames, el protagonista adquiere un rol y estatus definido, abordando la noticia de una forma diferente. Con la participación de los internautas, se busca concienciar acerca de la barbarie de las guerras, el terrorismo, la problemática medioambiental, epidemiológico y económica (Bogost, Ferrari \& Schweizer, 2012). 
Figura 8: Portada del newsgame Bacteria Salad.

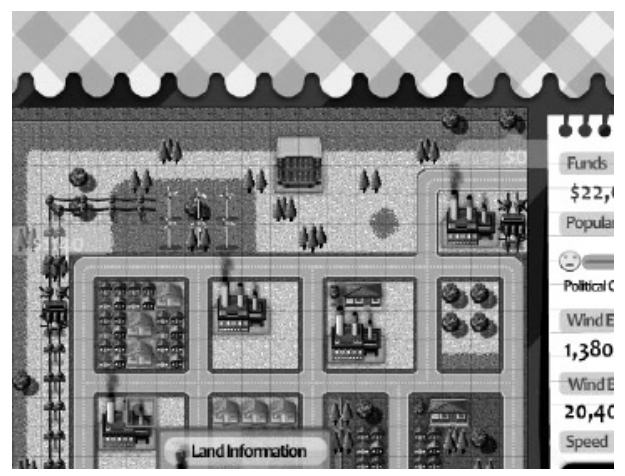

Fuente: Bogost, Ferrari \& Schweizer (2012).

Los newsgames no sólo se juegan: también implica el habla y la lectura. Para muchos, se pueden convertir en un modo de vida y de formación para el usuario. En estos se puede encontrar datos sobre política, historia, economía o sociología, aplicándose la experiencia cotidiana del jugador y así hacer posible el aprendizaje (Anneta, 2008).

\section{Metodología}

El análisis documental ${ }^{6}$ aborda una serie de documentos (en nuestro caso, newsgames ecuatorianos) con el propósito de representar qué se está haciendo en Ecuador con respecto a este campo. Se quiere conseguir que el artículo sea una fuente de referencia para los investigadores interesados en los newsgames en Ecuador. Asimismo, se realiza un proceso de interpretación y análisis de los newsgames ecuatorianos para, posteriormente, sintetizar los respectivos resultados.

En suma, la finalidad del presente artículo es ofrecer un análisis general de los newsgames, así como de la situación ecuatoriana, a fin que el contenido se configura como un instrumento de trabajo a futuro. En ese sentido, se han seleccionado dos newsgames ecuatorianos ( $\mathrm{La}$ Tortuga Maka y Tu Huella Ambiental) durante nuestro periodo de análisis (año 2017).

6 En el análisis documental se produce: $a$ ) un proceso de información ya que posibilita la recuperación de información para transmitirla de nuevo; $b$ ) un proceso de transformación, en el que los documentos primarios como son las páginas web van a ser analizados para convertirlo en un artículo de mayor acceso y difusión y c) un proceso analítico-sintético, porque los newsgames van a ser jugados, estudiados, interpretados y sintetizados para dar lugar a un artículo que los representan. 


\section{Resultados}

\subsection{La prensa digital y el videojuego en Ecuador}

Los primeros diarios digitales en Ecuador aparecieron en el año 1996, siendo los pioneros El Comercio y El Universo. Dos décadas más tarde, los diarios online no son el mayor ingreso económico de estos diarios. No obstante, cada vez más hay una mayor demanda de información por parte de los internautas, ya que estos solicitan videos, infografías, redes sociales, hipervínculos y newsgames.

Se cuenta con pocos datos acerca de los usuarios de videojuegos en Ecuador, pero existen asociaciones como Gamers Ecuador, compuesta por una población eminentemente masculina y joven. La industria del videojuego en Ecuador está aumentando y existen grandes nichos de inserción. Una de las limitaciones para el crecimiento en Ecuador se debe principalmente a que "la mayoría de programadores, que serían capaces de incursionar en esta actividad, no lo hacen por la falta de rentabilidad que esta presenta en el mercado local" (Puig, Parra \& Dreher, 2012, p. 33). El futuro es prometedor por la "gamificación"7.

En Ecuador se están estableciendo empresas relacionadas al videojuego y al desarrollo de soluciones de comunicación basadas en la interactividad como TrashBot Studios, Ximah y Blue Lizard Games. Esta última es una empresa multinacional canadiense, con clientes como Atari o Nintendo, que fue fundada en el 2008 por Nathalie Gauthier. Esta empresa es pionera en Ecuador en cuanto a la creación de videojuegos. Participa en eventos reconocidos e importantes a nivel mundial, como en la Conferencia Internacional de Desarrolladores de Juegos (Game Developers Conference, GDC). También se encuentra la empresa lojana Atixx (con su gerente David Eguiguren) que alcanzó el tercer lugar en la Global Game JAM 2013 (Sánchez Carpio, 2014).

En la actualidad, estas empresas no han creado newsgames, pero sí tienen potencial para hacerlo, ya que uno de los primeros pasos para entender la dinámica y objetivo de los newsgames es entender y conocer a los serious games. Ambos tienen objetivos similares, en cuanto a la comunicación y al periodismo. Además, tienen un carácter educativo y promueven una reflexión sobre determinado tema, aunque los serious games se enfoca en enseñar y los newsgames abordan temas y datos de actualidad (Ritterfeld, Cody \& Vorderer, 2009).

Uno de los juegos ecuatorianos, creado por Blue Lizard Games, es Nanoland (figura 9), en colaboración con María Fernanda Heredia, novelista ecuatoriana. Heredia escribe principalmente obras dirigidas a un público infantil, por lo que este juego toma ciertos extractos de las obras para educar a los niños de manera lúdica (Sánchez Carpio, 2014).

7 La gamificación (que tiene su raíz en el término game) consiste en la utilización de ciertas mecánicas de juego que intenta motivar y desarrollar aptitudes y habilidades en los jugadores. Aunque en un principio el concepto de la gamificación no fue concebido para videojuegos, así como tampoco para fines educativos, la popularización de Internet y los videojuegos está llamando la atención sobre las potencialidades de los videojuegos no solo de los programadores y desarrolladores, sino de psicólogos, sociólogos, educadores o comunicadores. 
Figura 9: Portada del newsgame Nanoland.

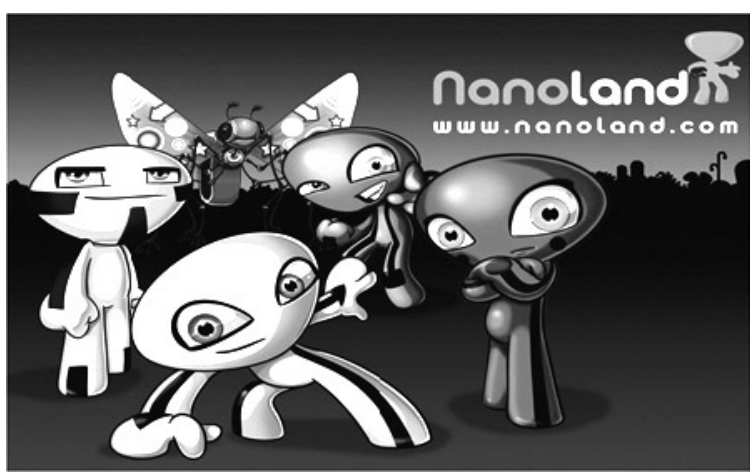

Fuente: Blue Lizard Games (2015).

\subsection{Newsgames para crear conciencia ambiental en Ecuador}

A diferencia de las temáticas globales de los newsgames, los diseñados en Ecuador se orientan al cuidado medioambiental. El sitio web www.quitoambiente.com, del Municipio de Quito, ha sido una de los primeras plataformas de información que implementa contenidos con juegos educativos en el país. Romina Carrasco, desarrolló uno de los juegos llamado Equilibroscopio (2009). Este juego se dirige básicamente a niños y consiste en crear seres primarios y secundarios en un "laboratorio de vida", manteniendo el equilibrio del ecosistema.

Su último proyecto, La Tortuga Maka (figura 10) lo realizó para el portal ecuatoriano La Hora. Este newsgame intenta concienciar a los jugadores acerca del peligro de las bolsas de plástico para la fauna silvestre en Galápagos. Al comienzo del juego aparece un personaje llamado "Reciclamán" que nos pide ayuda para salvar la vida de las tortugas marinas de Galápagos. El juego consiste en atravesar los obstáculos (basura, desperdicios). La meta es cambiar dicha situación, reduciendo el uso de plásticos y crear conciencia en el jugador. Este juego se vincula con Facebook y los usuarios pueden colgar fotos usando bolsas biodegradables, lo que les permite avanzar de nivel. 
Figura 10: Portada del newsgame La Tortuga Maka.

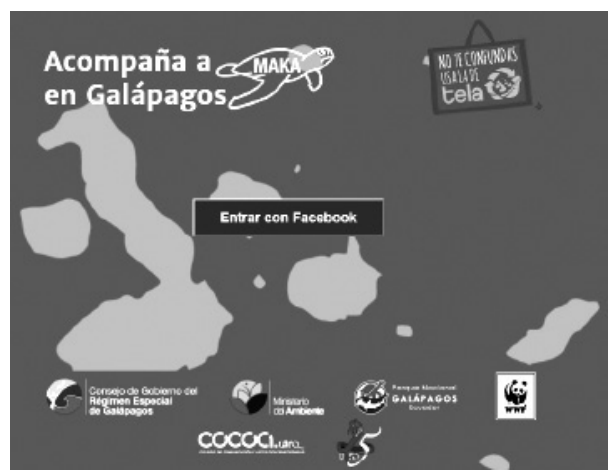

Fuente: Carrasco (2014).

Marcos Vaca, editor de diario El Comercio de Ecuador, participó en la realización de un newsgame llamado Tu Huella Ambiental $^{8}$ (figura 11). Exhibe un video de introducción reseñando sobre el cuidado y la importancia del medio ambiente. Luego, el internauta elige su género y comienza a utilizar diferentes opciones según sus acciones cotidianas de consumo: pañales desechables o de tela; cantidad de agua embotellada; si los empaques los desecha o los reutiliza, registrándose la basura producida. El newsgame posee unos gráficos básicos, pero cuenta con infografías explicativas. Al final del newsgame se indica qué tipo de huella ambiental ha dejado el jugador en el medio ambiente y cómo puede mejorar sus acciones ecológicas. Este juego brinda recompensas, reconocimiento, competencia, cooperación y altruismo.

Figura 11: Portada del newsgame Tu Huella Ambiental.

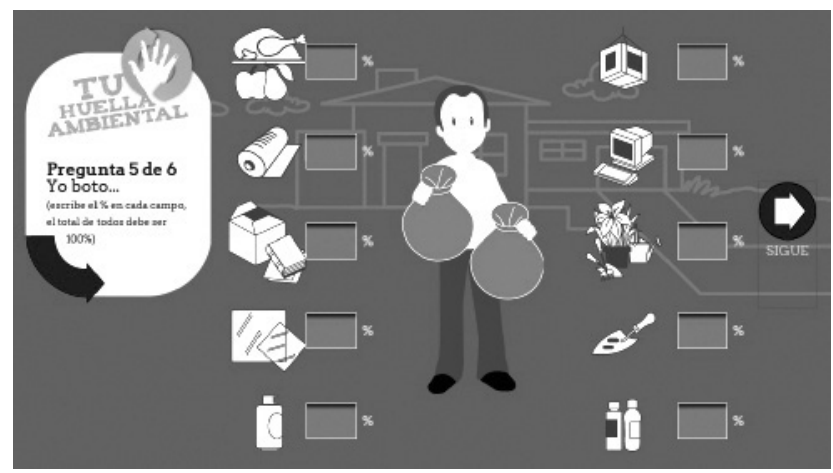

Fuente: Vaca (2012).

8 Tu Huella Ambiental fue un proyecto realizado en tres meses. Se creó por un equipo de ocho periodistas, tres diseñadores y un programador. Hasta la fecha, este ha sido el último newsgame del periódico. Los newsgames en Ecuador son más una apuesta experimental que una herramienta definida y enfocada al futuro. 
Los newsgames no tienen que ser complicados ni largos. Tampoco se requiere ser un programador experto o un ingeniero informático para desarrollar juegos sencillos que puedan estar vinculados a las noticias. Solo es necesario tener una buena asesoría y conceptos básicos de programación. Existen en Internet programas que permiten la descarga gratuita de plantillas de videojuegos ${ }^{9}$, donde se puede conseguir (por subasta) que un programador realice el newsgame ${ }^{10}$.

En los últimos años, el uso de Internet se ha intensificado, tanto para consumo como para publicación de contenidos comunicativos. Existe una reducción en la producción de libros físicos en Ecuador, pero está aumentando la difusión y consumo de los libros digitales. La cifra de internautas ecuatorianos va aumentando. Es por ello que los ecuatorianos leen más noticias en páginas web.

\subsection{Audiencias posibles y problemas de producción}

Los jóvenes consumidores de noticias están abandonando las noticias tradicionales a favor de las noticias “on demand". Estos citan específicamente la facilidad de educación y entretenimiento como factores que definen sus hábitos de consumo de noticias. A medida que los medios de comunicación buscan una forma de interactuar con esta audiencia más joven, esta muestra sus preferencias por los medios que quiere.

Claramente hay una audiencia posible para los newsgames. El entorno de juego permite a los usuarios explorar un evento de noticias de la manera que quieran: les permite interactuar y comprender una noticia en un entorno en el que están alfabetizados. De esta forma, los juegos basados en noticias tienen el potencial de cerrar la brecha entre el público (cansado de noticias tradicionales) y los proveedores de noticias (desesperados por conectarse con esta audiencia más joven).

Hay una serie de productores de contenido que podrían beneficiarse de la inclusión de newsgames. Dos elementos principales dan forma a la producción en línea en este entorno: a) la capacidad técnica de las salas de redacción para crear juegos basados en noticias y b) los cambios sociales y culturales necesarios para considerar los newsgames como un medio viable y apropiado para hacer noticias.

Los requisitos técnicos para crear juegos basados en noticias ya existen en las redacciones. Las aplicaciones de autoría de juegos (Macromedia's Flash) se utilizan ampliamente en

9 La plataforma en línea Coursera, por ejemplo, ofrece tipos de videojuegos de temática diferente: matemáticas, historia, ciencia y medio ambiente. Se pueden descargar y rediseñarlos como uno quiera.

10 El sitio web GetAcoder presenta proyectos de videojuegos que entran a formar parte de una subasta en línea donde desarrolladores de todo el planeta presentan ofertas y proponen precios para trabajar en las propuestas. Hay ofertas de juegos que pueden costar hasta 20000 dólares, así como también hay precios mínimos de 60 dólares si son sencillos (Mao, Capra, Harman \& Jia, 2015). 
salas de redacción en línea para crear galerías de audio y presentaciones interactivas. Actualmente, la producción de estos gráficos consume mucho tiempo, tanto por el nivel de investigación periodístico que requieren, como por la naturaleza altamente técnica de los programas utilizados para hacerlos. Los newsgames requerirán mucho más tiempo de producción, debido a la programación más compleja que debe escribirse.

La mayoría de las salas de redacción ejecutan un "ciclo de noticias". Una vez difundidas, las noticias pueden dejar de ser "nuevas" en cuestión de horas o minutos. En las noticias basadas en insumos de Internet, los plazos son ajustados y constantes. Los informes de noticias en línea, a menudo, están desactualizados tan pronto como se publican. Por estos motivos, como en el caso de los gráficos interactivos, los juegos basados en noticias se utilizarían mejor en el periodismo basado en temas de mayor duración o de investigación. Si bien habrá que abordar los problemas técnicos, la cultura actual de las salas de redacción y su tibia reacción ante las noticias en línea son motivo de mayor preocupación. Como señala Deuze (2001), el paso hacia la entrega de noticias en línea requiere un esfuerzo concertado y un cambio importante en la cultura del periodismo. De hecho, les ha tomado diez años a los operadores de medios ver los beneficios que traen las noticias online. Varias salas de redacción, incluyendo The New York Times, están ahora en el proceso de reorientación para enfatizar aún más los informes de Internet. Si bien la adición de newsgames sería un cambio menor, es probable que haya una resistencia significativa a la noción de un medio "frívolo", como los videojuegos que se utilizan para interactuar con las noticias.

\section{Conclusiones}

En el futuro, los newsgames podrían gozar de un papel más relevante. El uso de videojuegos (como método de entrega) podría abrir el ciberperiodismo hacia el espacio social, particularmente a través de las videoconsolas conectadas a la Internet. Estas nuevas plataformas de juego (PlayStation, de Sony; Xbox One, de Microsoft; Nintendo Wii U y Switch) podrían ser compatibles con servicios de noticias online. Así, los newsgames pueden ayudar a contextualizar y educar sobre esta nueva forma de utilizar los medios. Usar juegos para fomentar la comprensión de las noticias podría empoderar a más consumidores de noticias para que se involucren con esta nueva forma mediática.

Los juegos basados en noticias aún no dan un salto evolutivo que los equipare con otras plataformas online, pero tienen el potencial de agregar un contexto profundo a las noticias y relacionarse con los consumidores de noticias que buscan formas más interactivas y entretenidas de conectarse con las noticias. Para concretar esta posibilidad, los productores de newsgames deben ser cuidadosos a la hora de elegir el tema y la forma de retratarla. Los propios juegos de noticias se benefician con una visión abstracta o limitada de su tema, ya que intentar una realidad "documental" en un newsgame podría conducir a una posible parcialidad.

Tenemos precedentes históricos en las organizaciones de noticias que producen juegos para aumentar sus prácticas tradicionales de presentación. La industria de las cibernoticias ha avanzado en la presentación de información de forma no lineal (gráficos de noticias 
interactivos). Estos gráficos requieren de mucho trabajo, pero tienen la capacidad de proporcionar a sus usuarios un informe atractivo. Si bien existen grandes diferencias entre los juegos y los gráficos interactivos, los dos comparten características claves, como un entorno educativo (centrado en el usuario) que cobra todos los aspectos de las noticias.

Los juegos generalmente no son el mejor medio para informar las noticias de última hora. Esto se debe tanto al largo tiempo que lleva crearlos, como el dilema fundamental que consisten en permitir la libertad del jugador o reportar hechos verificados. En cambio, los juegos tienen la capacidad de "rodear" un informe de noticias para proporcionar un contexto más profundo y una mejor comprensión de los problemas subyacentes que intervienen en un problema o para explicar el funcionamiento de los procesos. El "estado ganador" de los newsgames debería ser una comprensión más profunda de un hecho noticioso.

El consumo de información está cambiando. Cada vez más usuarios se conectan a la red para informarse de una manera horizontal y no como (hasta el momento) de una manera vertical en donde los medios señalaban lo importante y lo que no lo era. Ecuador está experimentando nuevas formas de transmitir noticias. Sin embargo, los newsgames no son el recurso habitual: probablemente exista una condición cultural de cambio de paradigma y de consumo que no permite la masificación de este nuevo enfoque. No podemos descartar que en el futuro se expanda más. Se cuenta con algunas propuestas, pero todavía queda mucho camino por recorrer.

Con la nueva generación de los millennials, solo es cuestión de tiempo de que los cambios propuestos se produzcan y se plasmen en la realidad. En la actualidad, en Ecuador los serious games y los newsgames no han tenido la popularidad que se desearía. El juego de La Tortuga Maka, por ejemplo, tuvo pocos usuarios. En cambio, Tu Huella Ambiental tuvo una gran acogida y fue novedoso, pero no existe un seguimiento ulterior de ambas experiencias. La conclusión sería que no existe un compromiso serio (a largo plazo) por parte de los medios de comunicación ecuatorianos que les permita aceptar a los newsgames como productos en sus plataformas.

\section{Referencias}

Aarseth, E. (2007). Doors and perception: Fiction vs. simulation in games. Intermediality: History and Theory of the Arts, Literature and Technologies, 9, 35-44. doi: 10.7202/1005528ar

Anim-X. (2001). Majestic [PC download]. Activision.

Anneta, L. (2008). Video Games in Education: Why they should be used and how they are beingused. Theoryinto Practice,3(47),229-239. doi: 10.1080/00405840802153940

Archbold, J. (2014). The myth of the superhero (reseña de libro). Media International Australia, Incorporating Culture \& Policy, 150, 180-181. 
Arrobo, J., Ruiz, J., Hinojosa, M. \& Marín, I. (2017). Periodismo transmedia en España. Una oportunidad ante la crisis. En VV. AA. I Congreso Internacional de Periodismo Convergencias mediáticas y nueva narrativa latinoamericana (pp. 97-107). Quito: CIESPAL. Recuperado de https://goo.gl/T5DBLc

Blue Lizzard Games (2015). Nanoland [juego online]. Recuperado de http://www. nanoland.com/

Bogost, I., Ferrari, S. \& Schweizer, B. (2012). Newsgames: Journalism at play. Massachusetts: MIT Press.

Burton, J. (2005). News-Game Journalism: History, Current Use and Possible Futures. Australian Journal of Emerging Technologies and Society, 2(3), 87-99. Recuperado de https://goo.gl/JwNrDW

Carney, S., Eggertsson, S. \& Doret, M. (2009). Cutthroat capitalism: An economic analysis of the Somali pirate business model. Wired Magazine, 7(17). Recuperado de https://goo.gl/PwiZJC

Carrasco, R. (2014). Acompaña a Maka en Galápagos [juego online]. La Hora Noticias. Recuperado de http:/www.horadelplanetaecuador.org/appwwf/

Chan, E. \& Vorderer, P. (2006). Massively Multiplayer Online Games. En P. Vorderer \& J. Bryant (Eds.), Playing video games: Motives, responses, and consequences (pp. 77-88). Nueva Jersey: Lawrence Erlbaum Associates.

Congreso Internacional de Marketing, Publicidad y Comunicación (15 de agosto de 2018). CONMARK 2018 - Conferencia - Dr. Isidro Marín [video de YouTube]. Recuperado de https://goo.gl/KQY5BL

Creative Assembly (2004). Rome: Total War [PC CD-ROM]. Activision.

Deuze, M. (2001). Online Journalism: Modelling the First Generation of News Media on the World Wide Web. First Monday, 6(10). doi: https://doi.org/10.5210/ fm.v6i10.893

Domínguez Martín, E. (2014). Periodismo inmersivo: La influencia de la realidad virtual $y$ del videojuego en los contenidos informativos. Barcelona: Editorial UOC.

Eighty Dimensional Software (2004). Prime Minister Forever [PC download]. Eighty Dimensional Software.

Engel, M. (1996). Tickle the Public: One Hundred Years of the Popular Press. Londres: Gollancz. 
Frasca, G. (2000). Ephemeral Games: Is it Barbaric to Design Videogames after Auschwitz? En M. Eskelinen \& R. Koskimaa (Eds.), Cybertext Yearbook 2000. Jyvakyla: University of Jyvakyla.

Frasca, G. (2009). Juego, videojuego y creación de sentido. Una introducción. Revista Internacional de Comunicación Audiovisual, Publicidad y Literatura, 7, 37-44. Recuperado de https://goo.gl/w719cE

Fullerton, T. (2008). Documentary games: Putting the player in the path of history. En Z. Whalen y L. Taylor (Eds.), Playing the past: Nostalgia in video games and electronic literature (pp. 215-238). Nashville: Vanderbilt University Press.

García, A. \& Rupérez, P. (2007). Aproximaciones al periodismo digital. Madrid: Ediciones Dykinson.

Gearbox (2005). Brothers in Arms: The Road to Hill 30 [PC DVD-ROM]. Activision.

Gómez, S. \& Navarro, N. (2013). Videojuegos e Información: Una aproximación a los newsgames españoles como nueva óptica informativa. ICONO14, 2(11), 3-21. Recuperado de https://goo.gl/6sbva7

Gómez, S. \& Cabeza, J. (2016). El discurso informativo de los newsgames: El caso Bárcenas en los juegos para dispositivos móviles. Cuadernos.info, 38, 137-148. doi: $10.7764 /$ cdi.38.593

Harkin, J. (2014). Newsgames: Journalism at play (reseña de libro). Media International Australia, Incorporating Culture \& Policy, 150, 181-182.

Hess, A. (2007). You don't Play, You Volunteer: Narrative Public Memory Construction in Medal of Honor: Rising Sun. Critical Studies in Media Communication, 4(24), 339-356. doi: 10.1080/07393180701567729

Hinojosa, M., Robayo, C. \& Maldonado, M. (2016). Los inicios en el periodismo digital. Conceptos y consejos básicos. Saarbrücken: Editorial Académica Española.

Hinojosa, M., Ruiz, F. \& Marín, I. (2015). La informática. Origen y desarrollo. EAC, 3 , 58-65. Recuperado de https://goo.gl/2fWcMf

Huizinga, J. (1949). Homo ludens: A Study of the Play-Element in Culture. Londres: Routledge \& Kegan Paul. Recuperado de Recuperado de https://goo.gl/3mXgkX

Hutchison, D. (2007). Video games and the pedagogy of place. The Social Studies, 98, 35-40. doi: 10.3200/TSSS.98.1.35-40 
Infinity Ward (2003). Call of Duty [PC CD-ROM]. Activision.

James, J. (2017). Newsgames-Journalism Innovation through Game Design. American Journalism, 34(3), 379-381. doi: 10.1080/08821127.2017.1344074

López Pérez, B. (2013). Dimensiones narrativas e imaginarios para las imágenes de los MMORPG (Massive Multiplayer Online Rol-Playing Games) [tesis doctoral]. Universidad Autónoma Metropolitana, Azcapotzalco, México. Recuperado de http://hdl.handle.net/11191/5640

Mancera Rueda, A. (2009). La teoría de los géneros periodísticos en España: notas sobre su origen y estado de la cuestión. Sala de Prensa, 5(117), 1-25. Recuperado de https://goo.gl/jh4JSX

Mao, K., Capra, L., Harman, M. \& Jia, Y. (2015). Survey of the use of crowdsourcing in software engineering. Research Note, 15(01). Recuperado de https://goo.gl/ EoGNhw

Marín-Gutiérrez, I., Díaz-Pareja, E. \& Aguaded, I. (2013). La competencia mediática en niños y jóvenes: La visión de España y Ecuador. Chasqui. Revista Latinoamericana de Comunicación, 124, 43-49. Recuperado de https://goo.gl/rBXxkd

Martínez Cano, F. (2016). Newsgames: Nuevas tendencias en el periodismo ibérico. Recuperado de https://goo.gl/Jtsrn7

Noci, J. \& Salaverría, R. (2003). Manual de redacción ciberperiodística. Madrid: Grupo Planeta.

Paíno, A. \& Rodríguez, M. (2016). Renovarse o morir. Los “docugames”, una nueva estrategia transmedia que reinventa las formas de transmitir la realidad. ICONO14, $14,155-180$.

Puig, R., Parra, S. \& Dreher, M. (2012). Estudio de Mercado Servicio Desarrollo de Videojuegos en Ecuador. Estudio Comercial de la empresa ProChile y la Universidad Casa Grande. Guayaquil: Prochile. Recuperado de https://goo.gl/ iZiKbk

Raessens, J. (2006). Reality play: Documentary computer games beyond fact and fiction. Popular Communication, 4(3), 213-224. doi: 10.1207/s15405710pc0403_5

Raven Software (2001). Return to Castle Wolfenstein [PC CD-ROM]. Activision.

Ritterfeld, U., Cody, M. \& Vorderer, P. (Eds.). (2009). Serious games: Mechanisms and effects. Nueva York: Routledge. 
Sánchez Carpio, P. (2014). Newsgames como futura herramienta del periodismo en Ecuador análisis a partir de propuestas de juegos serios y nuevos formatos digitales periodísticos en el país (tesis de licenciatura). Universidad San Francisco de Quito, Ecuador.

Scolari, C. (2013). Homo Videoludens 2.0. De Pacman a la gamification. Barcelona: Universitat de Barcelona.

Squire, K. \& Steinkuehler, C. (2005). Meet the gamers. Library journal, 7(130), 38-41. Recuperado de https://goo.gl/Gvgbhx

Traffic Management (2004). JFK Reloaded [PC download]. Traffic Management lt.

Treanor, M., Mateas, M. \& Wardrip-Fruin, N. (2010). Kaboom! is a Many-Splendored Thing: An interpretation and design methodology for message-driven games using graphical logics. Recuperado de https://goo.gl/bgfjms

Vaca, M. (2012). Tu huella ambiental [juego online]. El Comercio. Recuperado de https:// goo.gl/9HXcku

Valderrama-Ramos, J. (2011). Videojuegos y educación: explorando aprendizajes entre adolescentes (tesis doctoral). ITESO-Universidad Jesuita de Guadalajara, México.

Valero Sancho, J. (2008). La infografía digital en el ciberperiodismo. Revista Latina de Comunicación Social, 63, 492-504. Recuperado de https://goo.gl/Yn9Ztn

Wolpaw, E. (2001). Medal of Honor: Allied Assault [PC CD-ROM]. Recuperado de https://goo.gl/29MK3W 